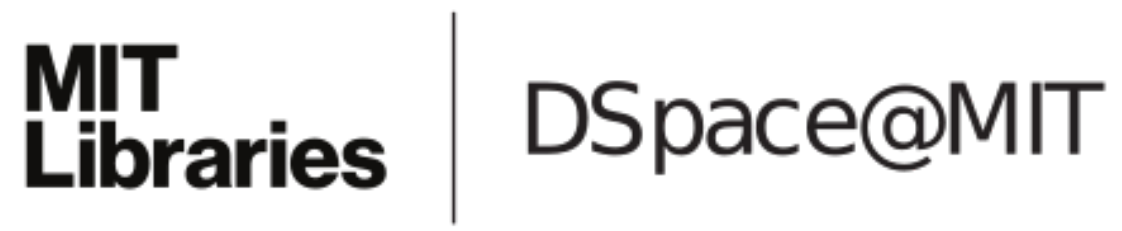

\author{
MIT Open Access Articles
}

Realization of a bipolar atomic Solc filter in the cavity-QED microlaser

The MIT Faculty has made this article openly available. Please share how this access benefits you. Your story matters.

Citation: Seo, Wontaek. et al. "Realization of a biopolar atomic Solc filter in the cavity-QED microlaser." Physical Review A 81.5 (2010)" 053824. (C) 2010 The American Physical Society

As Published: http://dx.doi.org/10.1103/PhysRevA.81.053824

Publisher: American Physical Society

Persistent URL: http://hdl.handle.net/1721.1/58732

Version: Final published version: final published article, as it appeared in a journal, conference proceedings, or other formally published context

Terms of Use: Article is made available in accordance with the publisher's policy and may be subject to US copyright law. Please refer to the publisher's site for terms of use. 


\title{
Realization of a bipolar atomic Šolc filter in the cavity-QED microlaser
}

\author{
Wontaek Seo, ${ }^{1}$ Hyun-Gue Hong, ${ }^{1}$ Moonjoo Lee, ${ }^{1}$ Younghoon Song, ${ }^{1}$ Young-Tak Chough, ${ }^{2}$ Wonshik Choi, ${ }^{3}$ C. Fang-Yen, ${ }^{3}$ \\ R. R. Dasari, ${ }^{3}$ M. S. Feld, ${ }^{3}$ Jai-Hyung Lee, ${ }^{1}$ and Kyungwon An ${ }^{1, *}$ \\ ${ }^{1}$ School of Physics and Astronomy, Seoul National University, Seoul 151-747, Korea \\ ${ }^{2}$ Department of Optical Communications Engineering, Gwangju University, Gwangju 503-703, Korea \\ ${ }^{3}$ G. R. Harrison Spectroscopy Laboratory, Massachusetts Institute of Technology, Cambridge, Massachusetts 02139, USA
}

(Received 13 October 2009; published 18 May 2010)

\begin{abstract}
We report experimental realization of a rudimentary atomic Šolc filter, recently proposed by Hong et al. [Opt. Express 17, 15455 (2009)]. It is realized by employing a bipolar atom-cavity coupling constant in the cavity-QED microlaser operating with a $\mathrm{TEM}_{10}$ mode in a strong coupling regime. The polarity flip in the coupling constant dramatically changes the photoemission probability of a two-level atom relative to unipolar coupling, resulting in multiple narrow emission bands in the detuning curve of the microlaser mean photon number. The observed resonance curves are explained well by a two-step, three-dimensional, geodesic-like motion of the Bloch vector in the semiclassical limit.
\end{abstract}

DOI: 10.1103/PhysRevA.81.053824

PACS number(s): 42.50.Pq, 42.50.Gy, 42.55.-f

\section{INTRODUCTION}

The coherent interaction between atoms and the electromagnetic field of a cavity has drawn much attention due to both its fundamental importance in quantum optics [1-8] and its applicability in quantum information processing [9-12]. In most of those studies, the lowest order of Hermite-Gaussian mode $\left(\mathrm{TEM}_{00}\right)$ of a Fabry-Perot-type cavity has been the natural choice due to the simplicity of its mode profile compared to higher order modes.

In recent years, high-order transverse $\operatorname{TEM}_{L M}(L+$ $M>0$ ) modes have been introduced to utilize their spatial modulation for identifying the atom's position [13-17] or for achieving Stark-shift-free trapping of atoms inside a cavity [18], where the spatial amplitude modulation of the coupling constant is the feature of interest in the higher order modes. Although the coupling constant also switches its sign depending on the location of the atom, such a sign flip plays little role since the atom experiences too many cycles of excitation and spontaneous decay during the sign flips in those studies.

However, the sign flip of the atom-cavity coupling constant can have a profound impact on the atom-cavity interactions $[19,20]$. In order to appreciate what kind of effects it might have, let us consider the well-known periodic poling [21] in nonlinear optics. By periodically poling a nonlinear medium, the phase mismatch otherwise present between a generated field and the medium's nonlinear polarization can be actively compensated, and thus nonlinear process can be greatly enhanced [22]. Likewise, in the optical Šolc filter [23], a stack of wave plates with their fast axes alternating works as a narrow bandpass filter due to stringent phase-matching conditions on wavelength and field polarization.

Conversely, it has been recently shown that the polarity flipping of the cavity field in the atom-cavity system can induce a filtering action on atomic photoemission process, namely the atomic Šolc filter [24], with one-to-one correspondence with the optical Šolc filter in the context of quasi-phase matching. The atomic Šolc filter exhibits not only multiple

*kwan@phya.snu.ac.kr narrow photoemission bands for an initially excited intracavity atom but also enables very strong sub-Poisson photon statistics of the cavity field [24,25], making it a quite attractive system for nonclassical field generation.

In this article, we report the realization of a rudimentary atomic Šlc filter with a single polarity flip of a cavity field in the cavity-QED microlaser [5]. In this realization, initially inverted two-level atoms traverse a TEM $_{10}$ mode and interact with the cavity field in a strong coupling regime. We observed that the polarity flip of the atom-cavity coupling constant dramatically changes the photoemission probability of those atoms, resulting in multiple narrow resonances in the detuning curve of the microlaser output. Our results are explained well by a two-step, three-dimensional, geodesic-like motion of the Bloch vector in the semiclassical limit.

\section{EXPERIMENTAL SETUP}

Our experimental setup, depicted in Fig. 1, is similar to those of Refs. [26,27]. The main difference is that we employ a $\mathrm{TEM}_{10}$ mode of a high-finesse $\left(\sim 10^{6}\right)$ Fabry-Perot-type resonator in order to realize precise bipolar atom-cavity coupling with a phase difference of $\pi$ for cavity-traversing atoms. The resonator (cavity) mirror has a radius of $300 \mu \mathrm{m}$ and a radius of curvature of $10 \mathrm{~cm}$. The cavity length is $1.0 \mathrm{~mm}$, and the mode waist is $41 \mu \mathrm{m}$. The resonator Fresnel number is 114 and the mixed Fresnel number is 10 so that the effect of diffraction is negligible. The cavity decay rates $\gamma_{c} / 2 \pi$ (half width) are $70 \mathrm{kHz}$ for $\mathrm{TEM}_{00}$ modes and $115 \mathrm{kHz}$ for $\mathrm{TEM}_{10}$ modes. We use ${ }^{1} S_{0} \leftrightarrow{ }^{3} P_{1}$ transition of atomic barium ${ }^{138} \mathrm{Ba}$ (wavelength $\lambda=791.1 \mathrm{~nm}$ ) with a spontaneous emission rate (half width) of $\gamma_{a} / 2 \pi \approx 25 \mathrm{kHz}$ for the excited state. A supersonic beam of barium atoms out of an oven is collimated with a rectangular aperture, $250 \mu \mathrm{m}$ (wide) $\times 25 \mu \mathrm{m}$ (high) and is then excited by a pump beam via adiabatic-following inversion process at $300 \mu \mathrm{m}$ in front of the cavity mode. The velocity variance of atom is $\Delta v / v_{0}=0.3$, where $v_{0}$ is the most probable velocity $(\sim 800 \mathrm{~m} / \mathrm{s})$ and $\Delta v$ is the full width at half maximum of the velocity distribution. The cavity transit time of atom is about $0.1 \mu \mathrm{s}$, during which atomic and cavity decays are negligible. The atomic beam is tilted by an angle $\theta$ with 
(a)

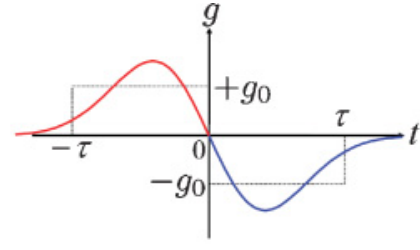

(b)

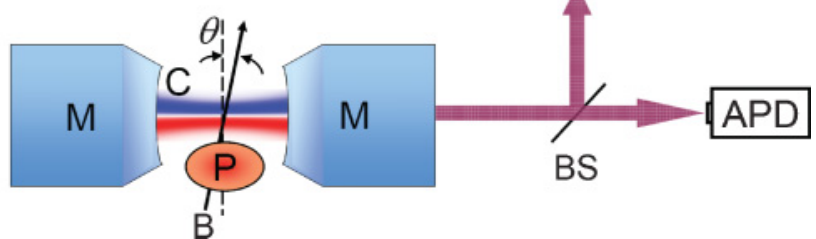

FIG. 1. (Color online) (a) Atom-cavity coupling $g(t)$ (solid line) that an atom experiences while traversing the $\mathrm{TEM}_{10}$ mode with a dotted line indicating an idealized flat-top profile with the same pulse area (for $t<0$ or $t>0$ ) as the actual TEM 10 mode. (b) Schematic for the experimental setup: $\mathrm{M}$, mirror; $\mathrm{C}$, cavity mode; $\mathrm{P}$, pump beam; $\theta$, atomic beam tilt angle; $\mathrm{B}$, barium atomic beam; $\mathrm{BS}$, beam splitter; APD, avalanche photo-diode for photon counting of the output signal; and $\mathrm{CCD}$, charge-coupled device for mode imaging.

respect to the normal incidence [dashed line in Fig. 1(b)]. The tilt angle is made large enough to achieve uniform atom-cavity coupling along the cavity axis [28,29] and also to prevent bichromatic interference between two traveling-wave components of atomic polarization [30]. The peak coupling constant $g$ associated with one traveling TEM $_{10}$ mode is $160 \mathrm{kHz}$ so that the strong coupling condition or the condition for avoided crossing [31], $g \gg\left|\gamma_{c}-\gamma_{a}\right| / 2$, is well satisfied in the experiment.

By virtue of a large transverse mode spacing of $6.6 \mathrm{GHz}$, we can easily select a group of modes with a specific value of $(L+M)$ out of many high-order transverse Hermite-Gaussian $\mathrm{TEM}_{L M}$ modes of the cavity. A small frequency difference $(\sim 5 \mathrm{MHz})$ is observed between the near-degenerate $\mathrm{TEM}_{10}$ and $\mathrm{TEM}_{01}$ modes. Their mode patterns imaged with a charge-coupled device (CCD) camera are shown in Fig. 2(b). They are rotated by about $24 \mathrm{deg}$ with respect to the atomic beam direction. We can selectively excite either of them by vertically adjusting the location of the aforementioned rectangular atomic-beam aperture whose vertical extension is only $30 \%$ of the cavity mode's full width.

\section{RESULTS AND DISCUSSIONS}

\section{A. Vertical scan for $L+M=1$ family}

In order to test the effectiveness of the bipolar coupling constant for realizing an atomic Šolc filter, we first measured the microlaser detuning curve, which is the mean number of photons $n$ in the cavity versus the cavity-atom detuning $\Delta$, for both $\mathrm{TEM}_{10}$ and $\mathrm{TEM}_{01}$ modes for various vertical positions of the atom-beam aperture as shown in Fig. 2. The average number of atoms in the cavity mode, determined from the fluorescence of ${ }^{1} S_{0} \leftrightarrow{ }^{1} P_{1}$ transition at $553.5 \mathrm{~nm}$, was $40( \pm 3)$. Because of the tilt angle $\theta$ of the atomic beam, we obtained two Doppler-shifted resonances in the detuning curve at $\omega=\omega_{0} \pm k v_{0} \theta$ [28-30], where $\omega$ and $\omega_{0}$ are the resonance frequencies of the cavity and the atom, respectively, with

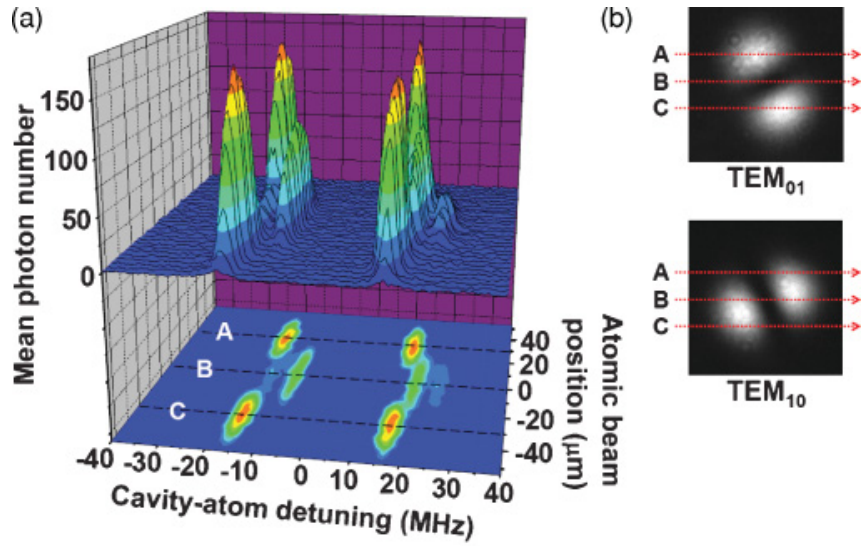

(c)

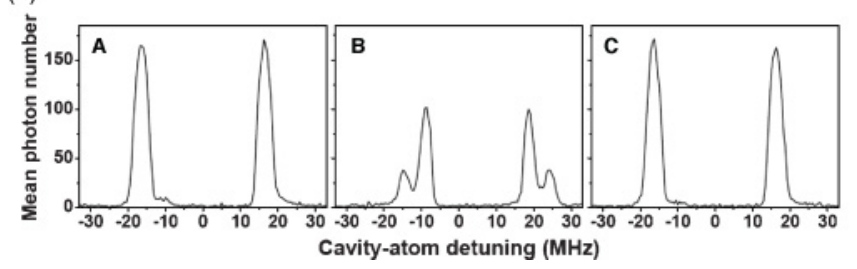

FIG. 2. (Color online) (a) Detuning curves of the microlaser output for various vertical positions of the atomic beam. (b) Transverse profiles of $\mathrm{TEM}_{01}$ and $\mathrm{TEM}_{10}$ modes when they are lasing, taken with a CCD camera. The cavity was locked at the maximum intensity of each mode. (c) The detuning curves at three atomic beam positions, $\mathrm{A}, \mathrm{B}$, and $\mathrm{C}$. For $\mathrm{A}$ and $\mathrm{C}$, the detuning curve for $\mathrm{TEM}_{01}$ mode is observed, whereas for $\mathrm{B}$, only the $\mathrm{TEM}_{10}$ mode undergoes lasing. Tiny shoulder-like structures on the right sides of each lasing peak of $\mathrm{TEM}_{01}$ mode are small contributions from the nearby (separated by $5 \mathrm{MHz}) \mathrm{TEM}_{10}$ mode.

$k=2 \pi / \lambda$. In Fig. 2, the value of the Doppler shift $k v_{0} \theta / 2 \pi$ is $16.3 \mathrm{MHz}$.

As the vertical position of the atomic beam varied, we found two regions (marked $\mathrm{A}$ and $\mathrm{C}$ ) where $\mathrm{TEM}_{01}$ mode undergoes lasing. These are qualitatively distinct from the region (marked B) where lasing by $\mathrm{TEM}_{10}$ mode is observed. For the $\mathrm{TEM}_{01}$ mode, a single peak appeared at each of the Dopplershifted resonance in the detuning curve due to the unipolar nature of the atom-cavity coupling along the trajectory of atoms, depicted by dotted lines $\mathrm{A}$ and $\mathrm{C}$ in Fig. 2(b). This identification of the atomic beam location across TEM $_{01}$ mode in the vertical direction is similar to what was demonstrated in Refs. [13,16,17]. The only difference is that a probe transmission is modified by the atom-cavity coupling in Refs. [13,16,17], whereas a coherent effect or lasing was used here. The region where TEM $_{10}$ undergoes lasing lies within $\pm 30 \mu \mathrm{m}$ around the mode center indicated by dotted line B. Note that the detuning curve there (B) shows two peaks around each Doppler-shifted resonance and the line width of each peak is noticeably smaller than that of $\mathrm{TEM}_{01}$ unipolar case (A and C) as in Fig. 2(c).

\section{B. Bloch sphere description}

This narrowed double-peak feature is in fact the indication of an atomic Šolc filter in action and can be understood in terms of the Bloch sphere picture [32] in the semiclassical limit $(n \gg 1)$. Suppose an equilibrium has been achieved and 
an atom initially inverted traverses the cavity. In the Bloch sphere picture, a state vector $\mathbf{R}$, initially pointing upward (i.e., fully excited), rotates around a torque vector $\Omega=(2 g \sqrt{n}, 0, \Delta)$ during an interaction time with $n$, the mean number of cavity photons in the equilibrium. The ground-state population, given by $\left(1-R_{z}\right) / 2$ after the interaction time, is then the probability that the atom emits a photon to the cavity during the passage or simply a gain function $G(n, \Delta)$ [26].

For simplicity, let us consider the idealized flat-top bipolar coupling constant as in Fig. 1(a). On resonance $(\Delta=0), \mathbf{R}$ rotates around $x$ axis of the Bloch sphere by $\Omega \tau$ due to $+g_{0}$ and then returns to the initial state due to $-g_{0}$. Thus, there is no net gain for lasing. At off-resonance, however, the torque vector with a nonzero detuning lies on the $x-z$ plane, and thus $\mathbf{R}$ follows a different path for $-g_{0}$ from the trace made by preceding $+g_{0}$ on the Bloch sphere. Consequently, the state vector ends up with a reduced $R_{z}$ from its initial value of unity and thus leads to a net lasing gain. The gain function $G(n, \Delta)$ in this case can be calculated analytically as [24]

$$
G(n, \Delta)=\frac{16 n g_{0}^{2} \Delta^{2}}{\left(4 n g_{0}^{2}+\Delta^{2}\right)^{2}} \sin ^{4}\left(\sqrt{4 n g_{0}^{2}+\Delta^{2}} \tau / 2\right),
$$

which is plotted in Fig. 3(b) as a function of a normalized detuning $\delta \equiv \Delta \tau$ and a normalized Rabi frequency $\eta \equiv 2 \sqrt{n} g_{0} \tau$.

\section{Maximum gain condition}

We can obtain conditions for a maximum gain $(G=1)$ by inspecting the motion of the state vector in the Bloch sphere. The maximum gain occurs when the final state vector points fully downward, which can be achieved in two steps as shown in Fig. 3(a). First, (i) the torque vector associated with $+g_{0}$
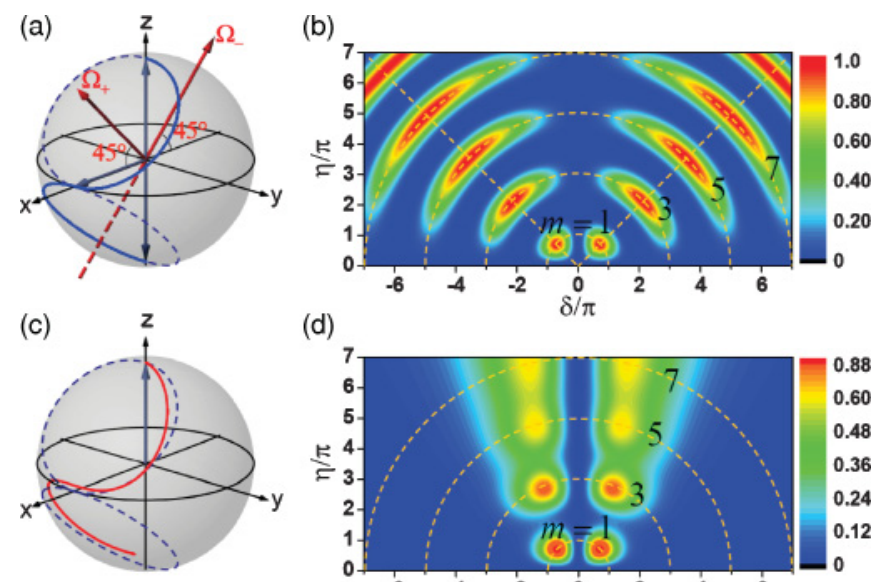

(d)

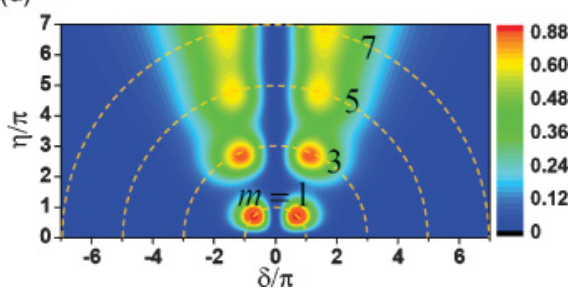

FIG. 3. (Color online) (a) In the Bloch sphere, the state vector (blue arrow), initially upward, can move in the downward direction by two successive rotations around a direction-switching torque vector (red arrow) for the bipolar coupling constant. (b) The gain function of Eq. (1) is plotted as a function of a normalized detuning $\delta$ and a normalized Rabi frequency $\eta$. (c) Actual Bloch vector trace. (d) The gain function accounting for the actual mode profile and the velocity distribution. The flat-top coupling constant $g_{0}$ is chosen to give the same $m=1$ gain peak conditions as the actual coupling constant. should be tilted from the $x$ axis by $\pi / 4$, and (ii) it should rotate the state vector exactly by an odd number of times of $\pi$ for the time interval $\tau$, thereby bringing $\mathbf{R}$ along the $x$ axis. For the next $\tau$, the torque vector is tilted by $3 \pi / 4$ with respect to the $x$ axis because of the sign flip of the coupling constant, and it rotates $\mathbf{R}$ again by the same odd number of times of $\pi$. These successive rotations in the Bloch sphere can bring the state vector fully downward.

These requirements are satisfied when (i) $|\Delta|=2 g_{0} \sqrt{n}$ and (ii) $\Omega \tau / \pi=m$ ( $m$ : odd integer). The condition (i) dictates two branch lines [orange dashed lines in Fig. 3(b)] at $\pm 45 \mathrm{deg}$ in $\delta-\eta$ space along which the maxima of $G$ are located. The condition (ii) dictates a branch circle with a radius $m \pi$ in $\delta-\eta$ space, and at its intersection with the branch lines, the gain maxima occurs. The presence of the multiple radii or the multiple layers of peaks is the consequence of coherent evolution or specifically multiple rotations of the state vector, which is also responsible for the multibranch solutions and quantum jumps between them in the cavity-QED microlaser [27,30]. Combining these two conditions, we obtain the detunings at which the gain maxima are located: $\Delta=$ $\pm m \pi /(\sqrt{2} \tau)$, which depends only on the interaction time. If we consider the actual atomic velocity distribution and the actual variation of the coupling constant of $\mathrm{TEM}_{10}$ mode, the shape of the gain function is smoothed out and the branch lines are curved inwardly, as shown in Fig. 3(d), although the gain peaks of $m=1$ are not affected much.

\section{Characteristics of detuning curves of $\mathrm{TEM}_{10}$}

To investigate in more detail the detuning curve of the $\mathrm{TEM}_{10}$ mode, we positioned the atomic beam aperture at the cavity center [dotted line B in Fig. 2(b)], where the node of $\mathrm{TEM}_{01}$ is located, in order to ensure almost no interaction with $\mathrm{TEM}_{01}$ mode and to induce near optimal bipolar interaction with TEM $_{10}$ mode. In Fig. 4(a), the resulting detuning curves grow as the number of the intracavity atoms is increased with a fixed Doppler shift of $k v_{0} \theta / 2 \pi=19.7 \mathrm{MHz}$. The separation between two peaks centered around a Doppler-shifted resonance is $8.0 \mathrm{MHz}$. The corresponding detunings are thus $\Delta / 2 \pi= \pm 4.0 \mathrm{MHz}$, which is consistent with the detunings for the maximum gain $\Delta_{0} / 2 \pi= \pm m /(2 \sqrt{2} \tau)= \pm 4.3 \mathrm{MHz}$ $(m=1)$. The small difference of $0.3 \mathrm{MHz}$ comes mainly from the assumption of a flat-model coupling instead of the actual amplitude-varying coupling. Since the Doppler-shifted zero resonance defers for different velocities, the gain does not vanish there when averaged over the atomic velocity distribution. The peak saturation at greater atom numbers then makes the peak-to-dip contrast further reduced. We also measured the separation between two peaks in another experiment, done with a slightly larger mean velocity of atoms, for various atomic beam tilt angles. We have confirmed that the two-peak separation has no dependence on the tilt angle, whereas the Doppler shift is proportional to it, as shown in Fig. 4(c).

The detuning curves of the TEM 10 -mode lasing in Fig. 4(a) is fit well by the semiclassical theory that includes the actual amplitude-varying coupling and the velocity distribution of the atomic beam. The height difference between peaks $A(D)$ and $\mathrm{B}(\mathrm{C})$ is mainly due to the velocity distribution of the atomic beam. For example, the locations of $\mathrm{A}$ and $\mathrm{B}$ are given 

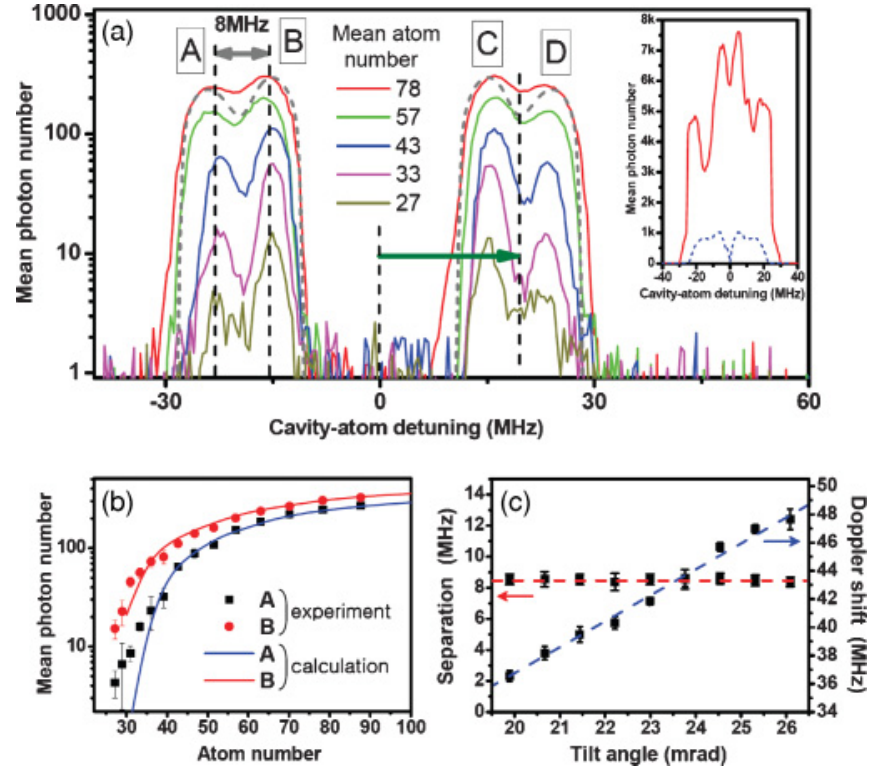

FIG. 4. (Color online) (a) Detuning curves of the microlaser mean photon number for the TEM 10 mode for various numbers of atoms $N$. The green and gray arrows indicate the Doppler shift of resonance and the two-peak separation, respectively. Inset: Quantum jumps to the $m=3$ layer solutions were observed with a large atom number at a small tilt angle $\left(k=10^{3}\right)$. An $m=1$ layer solution is also shown (blue dashed curve) for comparison. At this high gain, the two opposite traveling wave components become partially overlapped due to bichromatic interaction between them [30]. (b) The heights of peaks $\mathrm{A}$ and $\mathrm{B}$ in the detuning curve as the atom number. Grey dashed curve (for $N=78$ ) in (a) and solid lines in (b) are the fits by the semiclassical theory including the atomic velocity distribution and the actual mode profile. (c) The separation between peaks $\mathrm{C}$ and $\mathrm{D}$ versus the atomic beam tilt angle, measured with a slightly larger mean velocity of atoms than (a).

by $\Delta=-k v_{0} \theta \mp \pi v_{0} /(\sqrt{2} w)$, respectively, with $w$ as the effective mode waist in the flat-top model. When convoluted with the velocity distribution of $v_{0}$, peak $\mathrm{B}(\mathrm{C})$ is less broad and consequently higher than peak A(D). Moreover, the observed peak heights are significantly larger than the prediction by the semiclassical theory for small atom numbers $(<30)$ or for the photon number not much larger than unity since the photoemission by vacuum Rabi oscillation is neglected in the semiclassical theory.
As the atom number is increased, the growth of peaks A(D) and $\mathrm{B}(\mathrm{C})$ is slowed down. This saturation behavior of the mean photon number is essentially the same as the photon number stabilization observed in Refs. [27,30], accompanying sub-Poisson photon statistics [26]. Further increase of the atom number allows the next layer of gain peaks $(m=3)$ in Fig. 3(d) to come into action. Actually, when the mean atom number is further increased, quantum jumps to the next layer $(m=3)$ solutions were observed [the inset in Fig. 4(a)].

\section{E. Extending to higher order atomic Šolc filter}

The present experimental scheme can be extended to a high-order atomic Šolc filter by employing $\mathrm{TEM}_{L 0}$ with $L(>1)$ polarity flips. One can easily calculate the gain function for idealized flat-top multipolarity-switching modes, and the results show gain functions similar to Fig. 3(b) except that the number of branch lines is $L+1$ with the angle between them being $\pi /(L+1)$ [24]. In fact, the gain function has a one-to-one correspondence with the filter function of the optical folded Šolc filter [24] and is thus named the atomic Šolc filter. Moreover, the gain peaks on the first branch circle are squeezed so as to enable a strong gain-loss feedback at $\delta \sim \pm \pi$ [24], which is advantageous for nonclassical field generation.

\section{CONCLUSION}

In conclusion, we have demonstrated a rudimentary atomic Šolc filter by employing the bipolar atom-cavity coupling of $\mathrm{TEM}_{10}$ mode in the cavity-QED microlaser. The detuning curve of the microlaser output shows a double-peak structure around each Doppler-shifted resonance, and it can be well understood in terms of a two-step rotation of the Bloch vector around a direction-switching torque vector in the semiclassical limit. The present experimental scheme can be extended to the higher order Šolc filter to broaden the spectral range of laser gain further and to obtain the steeper gain-peak slope for nonclassical field generation.

\section{ACKNOWLEDGMENTS}

This work was supported by NRL and WCU grants from National Research Foundation of Korea.
[1] D. Meschede, H. Walther, and G. Müller, Phys. Rev. Lett. 54, 551 (1985).

[2] G. Rempe, H. Walther, and N. Klein, Phys. Rev. Lett. 58, 353 (1987).

[3] D. J. Heinzen and M. S. Feld, Phys. Rev. Lett. 59, 2623 (1987).

[4] R. J. Thompson, G. Rempe, and H. J. Kimble, Phys. Rev. Lett. 68, 1132 (1992).

[5] K. An, J. J. Childs, R. R. Dasari, and M. S. Feld, Phys. Rev. Lett. 73, 3375 (1994).

[6] J. J. Childs, K. An, M. S. Otteson, R. R. Dasari, and M. S. Feld, Phys. Rev. Lett. 77, 2901 (1996).
[7] J. McKeever, A. Boca, A. D. Boozer, J. R. Buck, and H. J. Kimble, Nature (London) 425, 268 (2003).

[8] S. Haroche and J. M. Raimond, Exploring the Quantum: Atoms, Cavities and Photons (Oxford University Press, Oxford, 2006).

[9] A. Kuhn, M. Hennrich, and G. Rempe, Phys. Rev. Lett. 89, 067901 (2002).

[10] J. McKeever, A. Boca, A. D. Boozer, R. Miller, J. R. Buck, A. Kuzmich, and H. J. Kimble, Science 303, 1992 (2004).

[11] K. M. Birnbaum, A. Boca, R. Miller, A. D. Boozer, T. E. Northup, and H. J. Kimble, Nature (London) 436, 87 (2005). 
[12] A. Kubanek, A. Ourjoumtsev, I. Schuster, M. Koch, P. W. H. Pinkse, K. Murr, and G. Rempe, Phys. Rev. Lett. 101, 203602 (2008).

[13] G. R. Guthöhrlein, M. Keller, K. Hayasaka, W. Lange, and H. Walther, Nature (London) 414, 49 (2001).

[14] P. Horak, H. Ritsch, T. Fischer, P. Maunz, T. Puppe, P. W. H. Pinkse, and G. Rempe, Phys. Rev. Lett. 88, 043601 (2002).

[15] P. Maunz, T. Puppe, T. Fischer, P. W. H. Pinkse, and G. Rempe, Opt. Lett. 28, 46 (2003).

[16] T. Puppe, P. Maunz, T. Fischer, P. W. H. Pinkse, and G. Rempe, Phys. Scr. T 112, 7 (2004).

[17] S. Nußmann, M. Hijlkema, B. Weber, F. Rohde, G. Rempe, and A. Kuhn, Phys. Rev. Lett. 95, 173602 (2005).

[18] T. Puppe, I. Schuster, A. Grothe, A. Kubanek, K. Murr, P. W. H. Pinkse, and G. Rempe, Phys. Rev. Lett. 99, 013002 (2007).

[19] R. Schlicher, Opt. Commun. 70, 97 (1989).

[20] A. Joshi and M. Xiao, J. Opt. Soc. Am. B 21, 1685 (2004).

[21] J. A. Armstrong, N. Bloembergen, J. Ducuing, and P. S. Pershan, Phys. Rev. 127, 1918 (1962).
[22] M. Houé and P. D. Townsend, J. Phys. D 28, 1747 (1995).

[23] I. Šolc, J. Opt. Soc. Am. 55, 621 (1965).

[24] H.-G. Hong, W. Seo, M. Lee, Y. Song, Y.-T. Chough, J.-H. Lee, and K. An, Opt. Express 17, 15455 (2009).

[25] L. Davidovich, Rev. Mod. Phys. 68, 127 (1996).

[26] W. Choi, J.-H. Lee, K. An, C. Fang-Yen, R. R. Dasari, and M. S. Feld, Phys. Rev. Lett. 96, 093603 (2006).

[27] C. Fang-Yen, C. C. Yu, S. Ha, W. Choi, K. An, R. R. Dasari, and M. S. Feld, Phys. Rev. A 73, 041802(R) (2006).

[28] K. An, R. R. Dasari, and M. S. Feld, Opt. Lett. 22, 1500 (1997).

[29] K. An, Y. T. Chough, and S. H. Youn, Phys. Rev. A 62, 023819 (2000).

[30] H.-G. Hong, W. Seo, M. Lee, Y. Song, W. Choi, C. Fang-Yen, R. R. Dasari, M. S. Feld, J.-H. Lee, and K. An, Phys. Rev. A 79, 033816 (2009).

[31] S.-B. Lee, J. Yang, S. Moon, S.-Y. Lee, J.-B. Shim, S. W. Kim, J.-H. Lee, and K. An, Phys. Rev. Lett. 103, 134101 (2009).

[32] R. P. Feynman, F. L. Vernon Jr., and R. W. Helwarth, J. Appl. Phys. 28, 49 (1957). 